\title{
Predicting 1-year cardiac transplantation survival using a donor-recipient risk-assessment tool
}

\author{
David L. Joyce, MD, ${ }^{a}$ Zhuo Li, MS, ${ }^{b}$ Leah B. Edwards, $\mathrm{PhD},{ }^{\mathrm{c}}$ Jon A. Kobashigawa, MD, ${ }^{\mathrm{d}}$ and \\ Richard C. Daly, MD
}

\section{ABSTRACT}

Objective: Many donor and recipient factors influence 1-year survival of patients after cardiac transplantation. To date, a statistical model has not been developed to assess the interplay of these factors in predicting outcomes, so we developed a risk-assessment tool to enhance decision-making.

Methods: We analyzed 29 variables that were reported in the United Network for Organ Sharing database for 24,540 cardiac transplantations performed between January 1, 2000, and June 30, 2015. For one half of the patients (the prediction population), a multivariable Cox regression model and the bootstrap resampling method were used to devise a scoring system predicting 1-year survival. The other half (the validation population) were stratified by score into 3 risk categories: high risk, medium risk, and low risk. One-year survival was compared among the 3 groups.

Results: Eleven variables were statistically significant in predicting 1-year survival. One-year survival for patients with risk scores of less than or equal to 8 , 9 to 15 , and greater than 15 were $91.2 \%, 81.7 \%$, and $64.6 \%$, respectively $(P<.001)$. The $\mathrm{C}$ index of the Cox regression model was calculated at 0.62 when using risk score as a continuous predictor.

Conclusions: Donor and recipient risk factors influence patient survival after cardiac transplantation. Long-term outcomes may be optimized with a statistically based risk model to improve donor-recipient matching. (J Thorac Cardiovasc Surg 2018;155:1580-90)

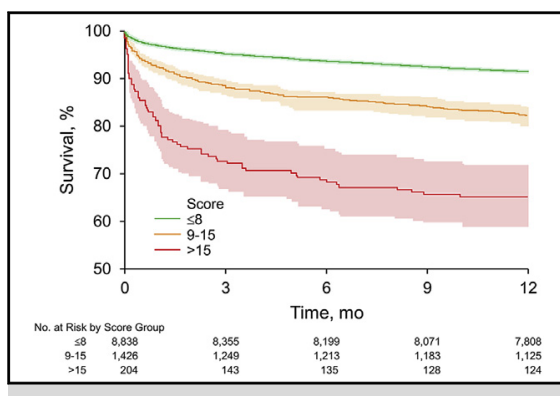

Quantitative risk assessment may enhance the process of donor-recipient pairing.

\section{Central Message}

Quantitative risk assessment may enhance the process of donor-recipient pairing.

\section{Perspective}

Existing risk-assessment tools in the field of cardiac transplantation fail to account for the interplay between donor and recipient characteristics. We developed a model to predict 1 -year posttransplant survival that can aid in the decision-making process.

See Editorial Commentary page 1591.
In the United States, the Centers for Medicare and Medicaid Services have identified 1-year survival as a key quality metric in the regulatory oversight of cardiac transplantation. ${ }^{1}$ Several donor- and recipient-specific risk factors have been identified as important predictors of poor 1-year survival. ${ }^{2-4}$ Risk models have been proposed to guide both donor organ selection and listing of recipient

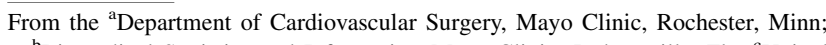
${ }^{\mathrm{b}}$ Biomedical Statistics and Informatics, Mayo Clinic, Jacksonville, Fla; ${ }^{\mathrm{c}}$ United Network for Organ Sharing, Richmond, Va; and ${ }^{\mathrm{d}}$ Cedars-Sinai Heart Institute, Los Angeles, Calif.

Read at the Annual Meeting of the International Society for Heart and Lung Transplantation, Washington, DC, April 27-30, 2016.

Received for publication Sept 29, 2016; revisions received Sept 6, 2017; accepted for publication Oct 10, 2017

Address for reprints: Richard C. Daly, MD, Division of Cardiovascular Surgery, Mayo Clinic, 200 First St SW, Rochester, MN 55905 (E-mail: rdaly@mayo.edu). $0022-5223 / \$ 36.00$

Copyright $₫ 2017$ Published by Elsevier Inc. on behalf of The American Association for Thoracic Surgery

https://doi.org/10.1016/j.jtcvs.2017.10.079 candidates. $^{5-10}$ However, the existing models have been limited in their ability to capture the interplay between donor and recipient risks when determining whether a given donor-recipient match is likely to result in 1-year survival. We sought to establish a scoring system that would enhance decision-making in determining whether an available donor organ would likely result in a successful 1-year outcome for a given recipient.

\section{METHODS}

Data from the Organ Procurement and Transplantation Network/United Network for Organ Sharing registry were used to select patients older than 18 years who underwent isolated cardiac transplantation between January

- Scanning this $\mathrm{QR}$ code will take you to a supplemental video for the article. 


\section{Abbreviations and Acronyms \\ $\mathrm{ECMO}=$ extracorporeal membrane oxygenation \\ LVAD $=$ left ventricular assist device}

1, 2000, and June 30,2015. An additional subset analysis was performed on patients who underwent transplant after 2008 to control for possible confounders due to changes in management (particularly the introduction of continuous-flow left ventricular assist device [LVAD] technology) that may have affected posttransplant outcomes. Those patients were included in the analysis. Risk factors for 1-year mortality were identified from a review of published literature. Variables were excluded from the analysis if data were missing for more than $15 \%$ of the overall study population. During analysis, patients with missing data on the specific variable of interest were excluded when the variable was analyzed. Patients were randomly assigned to 1 of 2 groups: 1 group was used for predicting 1-year survival outcome and building the scoring system (the prediction population), and the other group was used for evaluating the scoring system (the validation population). Patients were divided into 2 groups by sorting the patients' record numbers in numerical order and then assigning patients with an odd ranking order to the prediction data set and the ones with an even ranking order to the validation data set.

Cox regression models were used to identify the significant univariate and multivariable predictors of 1 -year mortality for the original prediction population. The proportional hazard assumption of the Cox regression model was tested for each predictive variable by testing the significance of the interaction terms between the variable and follow-up time in the Cox regression model predicting 1-year mortality. The bootstrap method was used to resample the prediction population 100 times with replacement, and the number of patients in each resampled data set was set to be the same number as the sample size of the original prediction population. For each resampled population, a multivariable model was selected among the significant predictors from univariate analysis with the stepwise selection method. Finally, only the variables that were entered into the multivariable model at least 70 times were included in the scoring system. The mean coefficients of all the multivariable models from the resamples were calculated and used to derive the prediction score. The predictor with the smallest coefficient was identified, and with its score set to 1, all the other predictors were assigned a score value equal to the quotient of their linear coefficients divided by the smallest coefficient. A score was then calculated for each patient in the validation population according to the scoring system generated previously. To generate low-risk (survival rate about $90 \%$ ), intermediate-risk (survival rate about $80 \%$ ), and highrisk (survival rate $<70 \%$ ) groupings, patients' scores were divided into 3 groups $(\leq 8,9-15$, and $>15)$ based on the 1-year survival rate of patients with each score. The Kaplan-Meier method was used to generate survival curves and calculate 1-year survival statistics for the 3 groups. The $\mathrm{C}$ index was calculated to measure the discrimination of the scores, and the calibration plot was drawn to show the agreement between the observed and predicted survival rates by grouping patients by every 10th percentile of the predicted risk of 1-year mortality. Descriptive statistics for categorical variables were summarized as frequency and percentage; continuous variables were summarized as mean (standard deviation) or median (range) as appropriate. All statistical tests were 2-sided, with an $\alpha$ level of .05 for statistical significance. Statistical analysis was performed with SAS version 9.4 software (SAS Institute Inc, Cary, NC).

\section{RESULTS}

A total of 24,540 patients older than 18 years underwent isolated cardiac transplantation between January 1, 2000, and June 30, 2015. This population was randomly divided into a prediction data set $(\mathrm{n}=12,270)$ and a validation data set $(n=12,270)$. Baseline and operative data were summarized for the 2 groups (Table 1). One-year survival for the prediction population was $88.2 \%$ (95\% confidence interval, $87.6 \%-88.8 \%$ ). After the exclusion of risk factors for which more than $15 \%$ of the data were missing for the study population, a total of 29 variables were selected for analysis, and no variable was missing more than $7 \%$ of the data. Donor-specific risk factors included distance from the transplant center, ischemic time, cause of death, left ventricular ejection fraction, hypertension, and clinical infection. Recipient-specific risk factors included dialysis, extracorporeal membrane oxygenation (ECMO), durable LVAD support, intra-aortic balloon pump, use of inotropes at listing, cause of heart failure, cerebrovascular disease, total bilirubin level, days on the waiting list, and number of previous transplants. Age, sex, ethnicity, presence of diabetes mellitus, and creatinine level were evaluated in both groups. Ethnic mismatch, sex mismatch, and donor-to-recipient heightweight ratio were studied as risk factors with a combined potential to influence 1-year survival. These variables were incorporated into a univariate Cox proportional hazards regression model (Table 2) and multivariable Cox proportional hazards regression model (Table 3 ).

The bootstrap method was used to create resamples and identify variables that were entered into the multivariable model more than $70 \%$ of the time. According to these results, the following risk factors were included in the scoring system: waiting time, recipient creatinine level greater than $2.0 \mathrm{mg} / \mathrm{dL}$ or condition requiring dialysis, LVAD, ECMO, recipient age, recipient total bilirubin of at least $3.0 \mathrm{mg} / \mathrm{dL}$, donor age, ischemic time, and female donor or sex mismatch (Table 4). The multivariable model containing these factors had a $\mathrm{C}$ index of 0.64 .

Risk scores were calculated for 10,468 patients in the validation population in which all variables of interest were available. Calculated risk scores ranged from 0 to 32 (mean [standard deviation], 5.1 [3.9]) (Figure 1). Groupings were then assigned according to the following cutoffs: low risk (score $\leq 8$ ), medium risk (score, 9-15), and high risk (score $>15$ ). Survival curves for the 3 risk groupings are shown in Figure 2: Low-risk patients had a 91.2\% 1-year survival, medium-risk patients had an $81.7 \%$ 1-year survival, and high-risk patients had a $64.6 \%$ 1-year survival $(P<.001)$. For the subset of patients who underwent transplant after 2008, the results were similar, with 1-year survival rates of $92.3 \%, 84.6 \%$, and $72.1 \%$ for low-, medium-, and high-risk patients, respectively $(P<.001)$ (Figure 3).

High-risk scores were most frequently related to endorgan function, age (of donor and recipient), and LVAD use (Figure 4). Although ECMO use was a heavily weighted risk factor in the scoring system, it contributed to only a small percentage of the overall high-risk group. Recipient 
TABLE 1. Baseline and operative data for the prediction and validation groups

\begin{tabular}{|c|c|c|c|c|c|}
\hline Feature & $\begin{array}{c}\text { Prediction } \\
(\mathrm{n}=12,270)^{*}\end{array}$ & $\begin{array}{c}\text { Validation } \\
(\mathrm{n}=\mathbf{1 2 , 2 7 0})^{*}\end{array}$ & $\begin{array}{c}\text { Total } \\
(\mathrm{n}=\mathbf{2 4 , 5 4 0})^{*}\end{array}$ & $P$ value & $\begin{array}{c}\text { Standardized } \\
\text { difference }\end{array}$ \\
\hline Year of transplant & & & & .843 & 0.01 \\
\hline 2000-2004 & $4496(36.6)$ & $4521(36.8)$ & 9017 (36.7) & & \\
\hline $2005-2009$ & $4463(36.4)$ & $4419(36.0)$ & $8882(36.2)$ & & \\
\hline $2010-2013$ & $3311(27.0)$ & $3330(27.1)$ & $6641(27.1)$ & & \\
\hline Recipient age, y & & & & .6184 & 0.01 \\
\hline$<55$ & $5956(48.5)$ & $5917(48.2)$ & $11,873(48.4)$ & & \\
\hline$\geq 55$ & $6314(51.5)$ & $6353(51.8)$ & $12,667(51.6)$ & & \\
\hline Donor age, y & & & & .2544 & -0.01 \\
\hline$<55$ & $11,819(96.3)$ & $11,852(96.6)$ & $23,671(96.5)$ & & \\
\hline$\geq 55$ & $451(3.7)$ & $418(3.4)$ & $869(3.5)$ & & \\
\hline Donor/receiver sex match & & & & .0585 & 0.03 \\
\hline Same sex: male & $7266(59.2)$ & $7315(59.6)$ & $14,581(59.4)$ & & \\
\hline Same sex: female & $1536(12.5)$ & $1643(13.4)$ & $3179(13.0)$ & & \\
\hline Male recipient, female donor & $2000(16.3)$ & $1923(15.7)$ & $3923(16.0)$ & & \\
\hline Female recipient, male donor & $1468(12.0)$ & $1389(11.3)$ & 2857 (11.6) & & \\
\hline Previous transplant & & & & .731 & 0.00 \\
\hline No & $11,997(97.8)$ & $11,989(97.7)$ & $23,986(97.7)$ & & \\
\hline Yes & $273(2.2)$ & $281(2.3)$ & $554(2.3)$ & & \\
\hline Previous heart transplant & & & & .2845 & 0.01 \\
\hline No & $12,066(98.3)$ & $12,044(98.2)$ & $24,110(98.2)$ & & \\
\hline Yes & $204(1.7)$ & $226(1.8)$ & $430(1.8)$ & & \\
\hline Waiting time & & & & .6713 & 0.02 \\
\hline Missing & 15 & 18 & 33 & & \\
\hline$<6 \mathrm{mo}$ & $8393(68.5)$ & $8393(68.5)$ & $16,786(68.5)$ & & \\
\hline $6 \mathrm{mo}$ to $1 \mathrm{y}$ & $1882(15.4)$ & $1829(14.9)$ & $3711(15.1)$ & & \\
\hline $1-2 y$ & $1195(9.8)$ & $1238(10.1)$ & $2433(9.9)$ & & \\
\hline$>2 y$ & $785(6.4)$ & $792(6.5)$ & $1577(6.4)$ & & \\
\hline Distance, miles & & & & .655 & 0.01 \\
\hline $0-10$ & $2845(23.2)$ & $2865(23.3)$ & $5710(23.3)$ & & \\
\hline $11-100$ & $3713(30.3)$ & $3647(29.7)$ & $7360(30.0)$ & & \\
\hline$>100$ & $5712(46.6)$ & $5758(46.9)$ & $11,470(46.7)$ & & \\
\hline Ischemic time, $\mathrm{h}$ & & & & .4539 & 0.02 \\
\hline Missing & 623 & 587 & 1210 & & \\
\hline$<4$ & $9233(79.3)$ & 9199 (78.7) & $18,432(79.0)$ & & \\
\hline $4-6$ & $2290(19.7)$ & $2368(20.3)$ & $4658(20.0)$ & & \\
\hline$>6$ & $124(1.1)$ & $116(1.0)$ & $240(1.0)$ & & \\
\hline Donor ejection fraction, $\%$ & & & & .0828 & -0.02 \\
\hline Missing & 355 & 358 & 713 & & \\
\hline$\geq 60$ & $8240(69.2)$ & $8361(70.2)$ & $16,601(69.7)$ & & \\
\hline$<60$ & $3675(30.8)$ & $3551(29.8)$ & $7226(30.3)$ & & \\
\hline Donor height to recipient height ratio & & & & .2273 & -0.01 \\
\hline Missing & 10 & 11 & 21 & & \\
\hline $0.5-1$ & $6357(51.9)$ & $6262(51.1)$ & $12,619(51.5)$ & & \\
\hline $1-2$ & $5903(48.1)$ & $5997(48.9)$ & $11,900(48.5)$ & & \\
\hline Donor weight to recipient weight ratio & & & & .5029 & 0.01 \\
\hline Missing & 8 & 9 & 17 & & \\
\hline$<1$ & $6617(54.0)$ & $6564(53.5)$ & $13,181(53.7)$ & & \\
\hline $1-2$ & $5603(45.7)$ & $5663(46.2)$ & $11,266(45.9)$ & & \\
\hline$>2$ & $42(0.3)$ & $34(0.3)$ & $76(0.3)$ & & \\
\hline TCR IV inotropes & & & & .9471 & 0.00 \\
\hline 0 & $7797(63.5)$ & $7802(63.6)$ & $15,599(63.6)$ & & \\
\hline 1 & $4473(36.5)$ & $4468(36.4)$ & $8941(36.4)$ & & \\
\hline
\end{tabular}


TABLE 1. Continued

\begin{tabular}{|c|c|c|c|c|c|}
\hline Feature & $\begin{array}{c}\text { Prediction } \\
(\mathbf{n}=\mathbf{1 2 , 2 7 0})^{*}\end{array}$ & $\begin{array}{c}\text { Validation } \\
(\mathbf{n}=\mathbf{1 2 , 2 7 0})^{*}\end{array}$ & $\begin{array}{c}\text { Total } \\
(\mathbf{n}=\mathbf{2 4 , 5 4 0})^{*}\end{array}$ & $P$ value & $\begin{array}{c}\text { Standardized } \\
\text { difference }\end{array}$ \\
\hline Recipient ethnicity & & & & .8503 & 0.02 \\
\hline White & $8865(72.2)$ & $8777(71.5)$ & $17,642(71.9)$ & & \\
\hline Black & $2100(17.1)$ & $2130(17.4)$ & $4230(17.2)$ & & \\
\hline Hispanic & $863(7.0)$ & $915(7.5)$ & $1778(7.2)$ & & \\
\hline Asian & $314(2.6)$ & $312(2.5)$ & $626(2.6)$ & & \\
\hline American Indian/Alaska Native & $37(0.3)$ & $37(0.3)$ & $74(0.3)$ & & \\
\hline Native Hawaiian/other Pacific Islander & $28(0.2)$ & $33(0.3)$ & $61(0.2)$ & & \\
\hline Multiracial & $63(0.5)$ & $66(0.5)$ & $129(0.5)$ & & \\
\hline Donor ethnicity & & & & .8455 & 0.02 \\
\hline Missing & 3 & 1 & 4 & & \\
\hline White & $8293(67.6)$ & $8298(67.6)$ & $16,591(67.6)$ & & \\
\hline Black & $1721(14.0)$ & $1745(14.2)$ & $3466(14.1)$ & & \\
\hline Hispanic & $1903(15.5)$ & $1905(15.5)$ & $3808(15.5)$ & & \\
\hline Asian & $192(1.6)$ & $187(1.5)$ & $379(1.5)$ & & \\
\hline American Indian/Alaska Native & $65(0.5)$ & $53(0.4)$ & $118(0.5)$ & & \\
\hline Native Hawaiian/other Pacific Islander & $17(0.1)$ & $18(0.1)$ & $35(0.1)$ & & \\
\hline Multiracial & $76(0.6)$ & $63(0.5)$ & $139(0.6)$ & & \\
\hline Ethnic mismatch & & & & .5967 & 0.01 \\
\hline Missing & 3 & 1 & 4 & & \\
\hline No & $6918(56.4)$ & $6878(56.1)$ & $13,796(56.2)$ & & \\
\hline Yes & $5349(43.6)$ & $5391(43.9)$ & $10,740(43.8)$ & & \\
\hline TCR cerebrovascular disease & & & & .6759 & -0.01 \\
\hline Missing & 170 & 208 & 378 & & \\
\hline 0 & $11,561(95.5)$ & $11,538(95.7)$ & $23,099(95.6)$ & & \\
\hline 1 & $539(4.5)$ & $524(4.3)$ & $1063(4.4)$ & & \\
\hline Donor clinical infection & & & & .4647 & 0.01 \\
\hline Missing & 474 & 535 & 1009 & & \\
\hline 0 & $6677(56.6)$ & $6587(56.1)$ & $13,264(56.4)$ & & \\
\hline 1 & $5119(43.4)$ & 5148 (43.9) & $10,267(43.6)$ & & \\
\hline Donor hypertension & & & & .7026 & 0.00 \\
\hline Missing & 70 & 54 & 124 & & \\
\hline 0 & $10,600(86.9)$ & $10,634(87.0)$ & $21,234(87.0)$ & & \\
\hline 1 & $1600(13.1)$ & $1582(13.0)$ & $3182(13.0)$ & & \\
\hline Recipient diabetes mellitus & & & & .152 & 0.02 \\
\hline Missing & 9 & 12 & 21 & & \\
\hline 0 & $9280(75.7)$ & $9181(74.9)$ & $18,461(75.3)$ & & \\
\hline 1 & $2981(24.3)$ & $3077(25.1)$ & $6058(24.7)$ & & \\
\hline Donor diabetes mellitus & & & & .6835 & -0.01 \\
\hline Missing & 45 & 36 & 81 & & \\
\hline 0 & $11,896(97.3)$ & $11,915(97.4)$ & $23,811(97.4)$ & & \\
\hline 1 & $329(2.7)$ & $319(2.6)$ & $648(2.6)$ & & \\
\hline ECMO at registration or transplant & & & & .004 & -0.04 \\
\hline No & $12,178(99.3)$ & $12,213(99.5)$ & $24,391(99.4)$ & & \\
\hline Yes & $92(0.7)$ & $57(0.5)$ & $149(0.6)$ & & \\
\hline TRR life support IABP & & & & .5703 & -0.01 \\
\hline 0 & $11,604(94.6)$ & $11,624(94.7)$ & $23,228(94.7)$ & & \\
\hline 1 & $666(5.4)$ & $646(5.3)$ & $1312(5.3)$ & & \\
\hline Thoracic diagnosis & & & & .4187 & 0.02 \\
\hline Idiopathic & $4056(33.1)$ & $4122(33.6)$ & $8178(33.3)$ & & \\
\hline Ischemic & $4698(38.3)$ & $4599(37.5)$ & 9297 (37.9) & & \\
\hline Other & $3516(28.7)$ & $3549(28.9)$ & $7065(28.8)$ & & \\
\hline
\end{tabular}

(Continued) 
TABLE 1. Continued

\begin{tabular}{|c|c|c|c|c|c|}
\hline Feature & $\begin{array}{c}\text { Prediction } \\
(\mathrm{n}=12,270)^{*}\end{array}$ & $\begin{array}{c}\text { Validation } \\
(\mathrm{n}=\mathbf{1 2 , 2 7 0})^{*}\end{array}$ & $\begin{array}{c}\text { Total } \\
(\mathrm{n}=\mathbf{2 4 , 5 4 0})^{*}\end{array}$ & $P$ value & $\begin{array}{l}\text { Standardized } \\
\text { difference }\end{array}$ \\
\hline Donor cardiac arrest & & & & .5703 & -0.01 \\
\hline Missing & 9 & 8 & 17 & & \\
\hline 0 & $11,660(95.1)$ & $11,680(95.3)$ & $23,340(95.2)$ & & \\
\hline 1 & $601(4.9)$ & $582(4.7)$ & $1183(4.8)$ & & \\
\hline Long-term use of device & & & & .5397 & -0.01 \\
\hline Missing & 809 & 764 & 1573 & & \\
\hline No & $7826(68.3)$ & $7900(68.7)$ & $15,726(68.5)$ & & \\
\hline Yes & $3635(31.7)$ & $3606(31.3)$ & $7241(31.5)$ & & \\
\hline Recipient total bilirubin, $\mathrm{mg} / \mathrm{dL}$ & & & & .0763 & 0.02 \\
\hline Missing & 485 & 516 & 1001 & & \\
\hline$<3$ & $11,128(94.4)$ & $11,035(93.9)$ & $22,163(94.2)$ & & \\
\hline$\geq 3$ & $657(5.6)$ & $719(6.1)$ & $1376(5.8)$ & & \\
\hline $\begin{array}{l}\text { Recipient creatinine }>2 \mathrm{mg} / \mathrm{dL} \text { or } \\
\text { receiving dialysis }\end{array}$ & & & & .4065 & -0.01 \\
\hline Missing & 412 & 444 & 856 & & \\
\hline 0 & $10,741(90.6)$ & $10,749(90.9)$ & $21,490(90.7)$ & & \\
\hline 1 & $1117(9.4)$ & $1077(9.1)$ & $2194(9.3)$ & & \\
\hline Donor cause of death & & & & .9178 & 0.01 \\
\hline Missing & 4 & 2 & 6 & & \\
\hline Anoxia & $1638(13.4)$ & $1634(13.3)$ & $3272(13.3)$ & & \\
\hline Cerebrovascular accident/stroke & $3027(24.7)$ & $2970(24.2)$ & $5997(24.4)$ & & \\
\hline Head trauma & $7255(59.1)$ & 7307 (59.6) & $14,562(59.4)$ & & \\
\hline CNS tumor & $113(0.9)$ & $114(0.9)$ & $227(0.9)$ & & \\
\hline Other & $233(1.9)$ & $243(2.0)$ & $476(1.9)$ & & \\
\hline
\end{tabular}

$T C R$, Transplant candidate registration; $I V$, intravenous; $E C M O$, extracorporeal membrane oxygenation; $T R R$, transplant recipient registration; IABP, intra-aortic balloon pump; $C N S$, central nervous system. *Categorical data are presented as number of patients (percentage of sample).

factors heavily influenced the score in terms of weighting and in terms of frequency within the high-risk population.

In the validation of this model, the $\mathrm{C}$ index of the Cox regression model was calculated at 0.62 when using risk score as a continuous predictor of 1-year mortality. When the scores were divided into 3 groups, the $\mathrm{C}$ index was 0.58 . The calibration plot in Figure 5 shows the agreement between the observed and the predicted 1-year survival rate.

\section{DISCUSSION}

Risk calculators in cardiac surgery have gained increasing popularity in recent years for quantifying and predicting major adverse outcomes after certain procedures. For coronary revascularization and valvular operations, the Society of Thoracic Surgeons has developed a widely used risk calculator based on more than 5.8 million surgical records in the Adult Cardiac Surgery Database. In contrast to decisions related to relatively routine types of cases, the decision related to accepting a donor heart for a given recipient is more complex and must account for the quality of the organ, the ability of the recipient to tolerate the transplant procedure, the long-term survival and quality-of-life benefit of transplanting the organ compared with alternative therapies, and the risk tolerance of the transplant center (which is often influenced by recent surgical outcomes). Unlike other solid-organ transplants (lung, kidney, and liver), heart allocation does not implement a measurement of predicted recipient outcomes. ${ }^{2}$ A further complication is that many of these decisions are made in a sleepdeprived state by the transplantation professional evaluating the donor at inopportune hours of the day or night. Not surprisingly, donor selection often fails to appropriately account for the actual risk associated with a given organ. ${ }^{11}$

We postulated that the process of donor assessment could be greatly enhanced by developing an improved risk model that would account for the interplay between donor, recipient, and combined risk factors in predicting 1-year survival. Using data from 24,540 cardiac transplantations entered into the Organ Procurement and Transplantation Network/United Network for Organ Sharing registry, we developed a risk-assessment tool that incorporates 9 different components into a scoring system that can be used to predict 1-year outcomes.

Comparable with results of previous studies, ${ }^{12,13}$ our results showed that donor age and ischemic time were highly predictive of 1-year outcomes. However, we found that the donor's distance from the transplant center did not affect 1-year survival. This finding suggests that additional factors that affect ischemic time (eg, case 
TABLE 2. Univariate Cox regression model predicting 1-year mortality

\begin{tabular}{|c|c|c|}
\hline Variable & HR $(95 \%$ CI $)$ & $P$ value \\
\hline \multicolumn{3}{|l|}{ Year of transplant } \\
\hline 2005-2009 vs $<2009$ & $0.81(0.72-0.91)$ & $<.001$ \\
\hline $2010-2013$ vs $<2009$ & $0.65(0.57-0.75)$ & $<.001$ \\
\hline Recipient age $\geq 55$ y vs $<55$ y & $1.24(1.12-1.38)$ & $<.001$ \\
\hline Donor age $\geq 55$ y vs $<55$ y & $1.80(1.45-2.23)$ & $<.001$ \\
\hline Female to female vs male to male & $1.13(0.96-1.32)$ & .145 \\
\hline Female to male vs male to male & $1.30(1.14-1.49)$ & $<.001$ \\
\hline Male to female vs male to male & $1.08(0.92-1.28)$ & .343 \\
\hline No. of previous transplants & $1.35(0.99-1.83)$ & .055 \\
\hline Previous heart transplant & $1.28(0.89-1.84)$ & .181 \\
\hline \multicolumn{3}{|l|}{ Waiting time } \\
\hline $6 \mathrm{mo}$ to $1 \mathrm{y} \mathrm{vs}<6 \mathrm{mo}$ & $1.14(0.99-1.32)$ & .068 \\
\hline $1-2$ y vs $<6$ mo & $1.08(0.91-1.29)$ & .390 \\
\hline$>2$ y vs $<6$ mo & $1.29(1.06-1.56)$ & .012 \\
\hline \multicolumn{3}{|l|}{ Distance } \\
\hline $11-100$ miles vs $\leq 10$ miles & $0.94(0.82-1.09)$ & .424 \\
\hline$>100$ miles vs $\leq 10$ miles & $1.02(0.89-1.16)$ & .806 \\
\hline \multicolumn{3}{|l|}{ Ischemic time } \\
\hline $4-6 \mathrm{~h}$ vs $<4 \mathrm{~h}$ & $1.41(1.25-1.60)$ & $<.001$ \\
\hline$>6 \mathrm{~h}$ vs $<4 \mathrm{~h}$ & $2.26(1.54-3.31)$ & $<.001$ \\
\hline Donor ejection fraction $<60 \%$ & $1.07(0.95-1.19)$ & .267 \\
\hline Donor height to recipient height ratio $1-2$ vs $<1$ & $1.09(0.98-1.21)$ & .105 \\
\hline \multicolumn{3}{|l|}{ Weight } \\
\hline Donor to recipient ratio $1-2$ vs $<1$ & $0.95(0.85-1.05)$ & .297 \\
\hline Donor to recipient ratio $>2$ vs $<1$ & $0.78(0.29-2.07)$ & .614 \\
\hline TCR IV inotropes at listing & $1.14(1.03-1.27)$ & .013 \\
\hline \multicolumn{3}{|l|}{ Recipient ethnicity } \\
\hline Black vs white & $1.20(1.05-1.37)$ & .007 \\
\hline Hispanic vs white & $0.95(0.77-1.17)$ & .637 \\
\hline Asian vs white & $1.02(0.73-1.42)$ & .909 \\
\hline American Indian/Alaska Native vs white & $1.26(0.52-3.02)$ & .612 \\
\hline Native Hawaiian/other Pacific Islander vs white & $0.61(0.15-2.45)$ & .489 \\
\hline Multiracial vs white & $0.69(0.29-1.66)$ & .406 \\
\hline \multicolumn{3}{|l|}{ Donor ethnicity } \\
\hline Black vs white & $1.00(0.86-1.17)$ & .951 \\
\hline Hispanic vs white & $1.02(0.88-1.18)$ & .801 \\
\hline Asian vs white & $1.16(0.79-1.72)$ & .448 \\
\hline American Indian/Alaska Native vs white & $0.79(0.36-1.77)$ & .573 \\
\hline Native Hawaiian/other Pacific Islander vs white & $0.00(0.00-0.00)$ & .922 \\
\hline Multiracial vs white & $1.02(0.53-1.97)$ & .946 \\
\hline Ethnic mismatch & $1.05(0.95-1.17)$ & .336 \\
\hline TCR cerebrovascular disease & $1.01(0.78-1.30)$ & .939 \\
\hline Donor clinical infection & $1.00(0.89-1.11)$ & .929 \\
\hline Donor history of hypertension & $1.23(1.07-1.42)$ & .004 \\
\hline Recipient diabetes mellitus & $1.06(0.94-1.19)$ & .349 \\
\hline Donor diabetes mellitus & $1.32(1.00-1.75)$ & .053 \\
\hline ECMO at registration or transplant & $3.72(2.62-5.28)$ & $<.001$ \\
\hline
\end{tabular}


TABLE 2. Continued

\begin{tabular}{llr}
\hline \multicolumn{1}{c}{ Variable } & HR $(\mathbf{9 5} \%$ CI $)$ & $P$ value \\
\hline TRR life support IABP & $1.32(1.07-1.62)$ & .008 \\
Thoracic diagnosis & & \\
$\quad$ Ischemic vs idiopathic & $1.30(1.14-1.47)$ & .001 \\
$\quad$ Other vs idiopathic & $1.22(1.07-1.40)$ & .004 \\
Donor cardiac arrest & $0.85(0.66-1.10)$ & $<.001$ \\
Recipient long-term use of device & $1.37(1.22-1.53)$ & $<.001$ \\
Recipient total bilirubin $\geq 3$ vs $<3 \mathrm{mg} / \mathrm{dL}$ & $2.14(1.80-2.55)$ & $<.001$ \\
Recipient creatinine $>2 \mathrm{mg} / \mathrm{dL}$ or receiving dialysis & $2.64(2.31-3.01)$ & \\
Donor cause of death & & $<.001$ \\
$\quad$ Cerebrovascular accident/stroke vs anoxia & $1.39(1.16-1.66)$ & .242 \\
Head trauma vs anoxia & $1.10(0.93-1.31)$ & .179 \\
CNS tumor vs anoxia & $1.42(0.85-2.37)$ & .303 \\
Other vs anoxia & $1.23(0.83-1.83)$ & \\
\hline
\end{tabular}

$H R$, Hazard ratio; $C I$, confidence interval; $T C R$, transplant candidate registration; $I V$, intravenous; $E C M O$, extracorporeal membrane oxygenation; $T R R$, transplant recipient registration; IABP, intra-aortic balloon pump; $C N S$, central nervous system.

complexity) may have an important role, which may be difficult to estimate by travel time alone. Donor risk factors such as race mismatch and renal impairment were not statistically significant in our prediction model, unlike their proposed value in previous publications.

Despite the increasing emphasis that is often placed on donor organ quality, recipient risk factors were much more predictive of a poor outcome in terms of their overall contribution to our scoring system. The Index for Mortality Prediction After Cardiac Transplantation score, described by Weiss and colleagues ${ }^{5,14}$ in 2011, identified and validated the utility of a score comprising 11 recipient risk factors in predicting 1-year mortality. Many of the variables of interest were relevant in our analysis as well (age, renal and liver function, and LVAD use). Nevertheless, it is interesting to note the degree to which evolving trends within the field of cardiac transplantation can alter the risk calculation even over a 5-year period. Increased use of LVAD and ECMO as bridging strategies has contributed to longer waiting times. These variables were significant contributors to our scoring system, even though they were not accounted for in the Index for Mortality Prediction After Cardiac Transplantation algorithm (aside from LVADs other than HeartMate II [Abbott Laboratories, Abbott Park, Ill]).

These findings also illuminate some of the consequences that are likely to occur with the introduction of a new allocation system. The proposed changes to this system will allocate organs at the highest priority level to patients who are supported with ECMO. Although only 27 patients underwent bridging with ECMO in the present analysis, these patients experienced a substantial increase in mortality (representing the highest risk variable among all the factors that were studied). Certainly it stands to reason that while these patients will benefit from an increase in the number of donor offers under the new allocation system, a thoughtful approach to accepting only an "ideal" organ may be warranted in light of these findings. Furthermore, for many patients, recipient optimization to improve endorgan function may very well become a prerequisite because many of these patients will have prohibitive risk scores even with a perfect donor organ.

The role of mechanical circulatory support in influencing posttransplant outcomes has been controversial since the concept of bridge to transplant was first introduced. Outcomes with LVAD support have progressively improved over the past 15 years, and this is likely accounted for by a combination of improved patient selection, better devices, and enhanced postoperative care. ${ }^{15}$ To minimize the degree to which these changes would diminish the utility of our risk model, our analysis excluded patients who received a transplant before January 1, 2000. Given the limited data on device type available in the registry, we selected an inclusive LVAD variable that combined all patients who were supported with a durable assist device before transplant.

The relationship between donor sex and posttransplant outcomes has been controversial in recent years. Institutional data from the Stanford University Medical Center showed a lower 1-year survival among male recipients of organs from female donors compared with male recipients of organs from male donors. ${ }^{16}$ Following on this work, authors from Stanford reported an analysis of 60,584 patients in the International Society for Heart and Lung Transplant registry, which showed a $10 \%$ survival benefit for male recipients of allografts from male donors compared with allografts from female donors and a $10 \%$ higher mortality rate among female recipients of allografts from female donors (compared with allografts from male donors). ${ }^{17}$ Other 
TABLE 3. Multivariable Cox regression model predicting 1-year mortality

\begin{tabular}{|c|c|c|c|}
\hline Risk factor & Hazard ratio & $95 \%$ CI & $P$ value \\
\hline \multicolumn{4}{|l|}{ Year of transplant } \\
\hline $2005-2009$ vs $<2009$ & 0.87 & $0.76-0.99$ & .036 \\
\hline $2010-2013$ vs $<2009$ & 0.66 & $0.57-0.77$ & $<.001$ \\
\hline \multicolumn{4}{|l|}{ Recipient age } \\
\hline$\geq 55$ y vs $<55$ y & 1.25 & $1.11-1.40$ & $<.001$ \\
\hline \multicolumn{4}{|l|}{ Donor age } \\
\hline$\geq 55$ y vs $<55$ y & 1.46 & $1.13-1.88$ & .003 \\
\hline \multicolumn{4}{|l|}{ Waiting time } \\
\hline 6-12 mo & 1.10 & $0.94-1.29$ & .229 \\
\hline $1-2$ y & 1.15 & $0.95-1.39$ & .153 \\
\hline$>2 y$ & 1.31 & $1.06-1.63$ & .013 \\
\hline $\begin{array}{l}\text { Recipient dialysis or } \\
\text { creatinine }>2.0 \mathrm{mg} / \mathrm{dL}\end{array}$ & 2.37 & $2.05-2.75$ & $<.001$ \\
\hline \multicolumn{4}{|l|}{ Ischemic time } \\
\hline $4-6 \mathrm{~h}$ vs $<4 \mathrm{~h}$ & 1.31 & $1.15-1.50$ & $<.001$ \\
\hline$>6 \mathrm{~h} \mathrm{vs}<4 \mathrm{~h}$ & 2.29 & $1.54-3.42$ & $<.001$ \\
\hline $\begin{array}{l}\text { Recipient total bilirubin } \\
\quad \geq 3.0 \mathrm{mg} / \mathrm{dL} \text { vs }<3 \mathrm{mg} / \mathrm{dL}\end{array}$ & 2.03 & $1.67-2.45$ & $<.001$ \\
\hline $\begin{array}{l}\text { Female to female vs male to } \\
\text { male }\end{array}$ & 1.22 & $1.02-1.46$ & .033 \\
\hline $\begin{array}{l}\text { Female to male vs male to } \\
\text { male }\end{array}$ & 1.29 & $1.11-1.51$ & $<.001$ \\
\hline $\begin{array}{l}\text { Male to female vs male to } \\
\text { male }\end{array}$ & 1.27 & $1.06-1.52$ & .010 \\
\hline Ethnic mismatch & 1.15 & $1.03-1.30$ & .016 \\
\hline ECMO & 3.12 & $2.09-4.66$ & $<.001$ \\
\hline LVAD & 1.39 & $1.23-1.58$ & $<.001$ \\
\hline $\begin{array}{l}\text { Ischemic vs idiopathic } \\
\text { diagnosis }\end{array}$ & 1.18 & $1.02-1.36$ & $<.001$ \\
\hline Other vs idiopathic diagnosis & 1.20 & $1.04-1.40$ & $<.001$ \\
\hline
\end{tabular}

$C I$, Confidence interval; ECMO, extracorporeal membrane oxygenation; $L V A D$, left ventricular assist device.

studies have questioned both the relevance of sex mismatch and the associated risk of hearts from female donors. ${ }^{18,19}$

In light of these observations, we combined donor sex and sex match into a unique sex match variable with 4 potential donor-recipient designations: male to male, female to female, male to female, and female to male. By treating male-to-male configurations as the reference point with the best outcomes, we determined the relative risk of the other configurations and included each of them in the risk model. Although sex matching was only a weak predictor of posttransplant survival (generating a score of 2 for all but maleto-male configurations), there is no question that these compatibility issues are important. The discordance between numerous studies (including ours) in describing the importance of sex matching on posttransplant outcomes suggests that this is an area in which further study is needed. It is unlikely that the methodology used in studies such as this one, which incorporate elements of a large database in performing this type of analysis, will be able to tease out the mechanisms behind these observations.

This study has important implications for the field of cardiac transplantation. By highlighting the importance of both recipient and donor factors in making decisions, this risk model allows for a balanced risk assessment that has the potential to improve organ matching. Given the complexity of this decision and the paucity of quantifiable metrics, this scoring system has the potential to decrease the incidence of poor 1-year outcomes after transplant and to increase the use of available donor organs by identifying suitable low-risk recipients. A comparable analogy exists in the field of kidney transplantation, where the Kidney Donor Profile Index was developed to improve organ allocation. ${ }^{20}$ Those data are now presented along with other donor characteristics as part of the DonorNet Web site.

From a practical standpoint, it is worth noting that of the 10,468 patients in the validation set, a total of $1630(15.6 \%)$ ultimately received a transplanted organ that, with our model, would have carried a predicted 1-year survival of less than $80 \%$. Although it is common to undertake treatment of such high-risk patients in the practice of cardiothoracic surgery, these sorts of risk models (much like the Society of Thoracic Surgeons Risk Calculator) provide an additional quantifiable basis for these decisions. Although a model like this will not substitute for experience and judgment, it may improve the dialogue between members of the transplant team-particularly when a center is faced with multiple, consecutive high-risk patients, which could threaten a program's outcomes.

Perhaps the best illustration of the utility of a predictive tool such as this comes from applying it to the everyday practice of a tertiary care academic facility. As with many transplant centers, we frequently evaluate "extreme-risk" donors. These include patients receiving ECMO and other forms of short-term mechanical circulatory support, patients with multiple reoperations from congenital heart disease, and patients awaiting multiorgan transplant (including liver before heart). Even though relatively few donor risks were found to be highly predictive of posttransplant outcomes in this model (namely, age and ischemic time), an accurate understanding of the combined risk is extremely useful in refining the process of donor selection for these patients. As an example, a 56-year-old patient supported with ECMO but who was otherwise in stable condition and had adequate end-organ perfusion would be an acceptable match with a young donor and an ischemic time of less than 4 hours. However, when considering older donors from greater distances (or other factors that could increase ischemic time), the transplant center would have to consider the predicted risk in light of recent Scientific Registry of 
TABLE 4. Risk score criteria

\begin{tabular}{|c|c|c|c|}
\hline Risk factor & $\begin{array}{c}\text { No. of times selected among } \\
100 \text { resamples }\end{array}$ & $\begin{array}{l}\text { Mean parameter } \\
\text { estimate }(95 \% \mathrm{CI})\end{array}$ & Score \\
\hline Waiting time 6 mo to $1 \mathrm{y}$ & 78 & $0.11(-0.05$ to 0.27$)$ & 1 \\
\hline Waiting time $1-2 \mathrm{y}$ & 78 & $0.18(-0.04$ to 0.40$)$ & 2 \\
\hline Waiting time $>2 \mathrm{y}$ & 78 & $0.36(0.15-0.56)$ & 3 \\
\hline Recipient dialysis or creatinine $>2.0 \mathrm{mg} / \mathrm{dL}$ & 100 & $0.86(0.71-1.00)$ & 8 \\
\hline ECMO & 99 & $1.14(0.70-1.59)$ & 10 \\
\hline Donor age $\geq 55 \mathrm{y}$ & 77 & $0.42(0.24-0.61)$ & 4 \\
\hline Recipient age $\geq 55 \mathrm{y}$ & 91 & $0.23(0.12-0.34)$ & 2 \\
\hline LVAD & 100 & $0.34(0.21-0.46)$ & 3 \\
\hline Male to female & 90 & $0.27(0.10-0.43)$ & 2 \\
\hline Female to male & 90 & $0.24(0.09-0.40)$ & 2 \\
\hline Female to female & 90 & $0.20(-0.01$ to 0.40$)$ & 2 \\
\hline Ischemic time $4-6 \mathrm{~h}$ & 100 & $0.26(0.11-0.41)$ & 2 \\
\hline Ischemic time $>6 \mathrm{~h}$ & 100 & $0.85(0.42-1.28)$ & 8 \\
\hline Recipient total bilirubin $\geq 3.0 \mathrm{mg} / \mathrm{dL}$ & 100 & $0.69(0.49-0.88)$ & 6 \\
\hline
\end{tabular}

$C I$, Confidence interval; $E C M O$, extracorporeal membrane oxygenation; $L V A D$, left ventricular assist device.

Transplant Recipients outcomes before accepting such an offer. In many situations, this predictive tool may appropriately restrict the range of acceptable donors to local hospitals, thereby improving 1-year outcomes not only for the high-risk recipients but also for patients lower on the waiting list who would be expected to have improved survival from a lower-quality donor organ.

As with all efforts to establish predictive models for mortality risk in the field of cardiac transplantation, our assessment tool is limited by the problem of missing data related to several factors that are widely believed to be important for posttransplant survival. Variables with missing data from more than $15 \%$ of the patients included recipient ventilator dependence, transpulmonary gradient, left ventricular hypertrophy, and panel-reactive antibodies. This

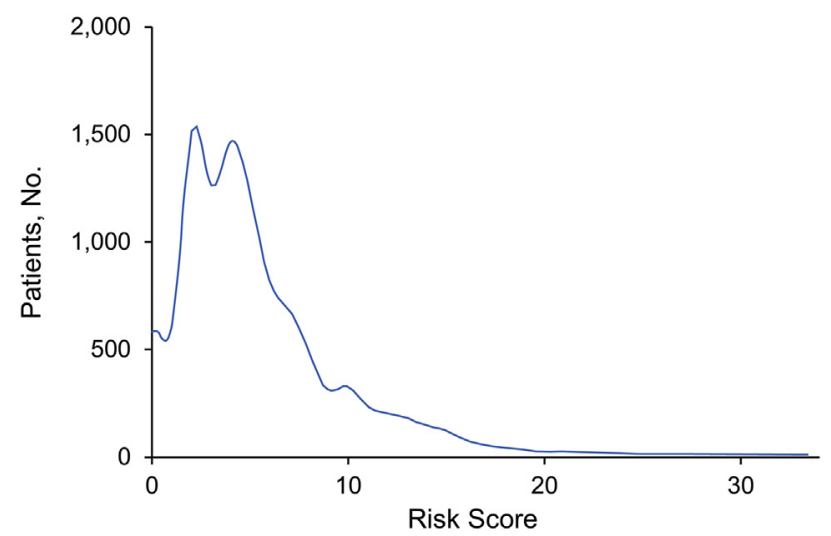

FIGURE 1. Distribution of risk scores. underscores the importance of meticulous data management and the need for routine updating to achieve the greatest degree of accuracy. It would have been ideal to adjust for center effect, but center information also was missing in the data set. We expect that the predictive ability of the model will be greatly enhanced as additional data elements become available for these important variables. In addition, given the missing data related to device type, we could not effectively determine the extent to which various forms of mechanical circulatory support influenced 1-year survival. Technologic evolution affects cardiac transplantation in various ways and challenges efforts to improve and revise risk models such as ours. Without question, these limitations at least in part account for the $\mathrm{C}$ index being lower than what one would ideally hope to achieve in a predictive

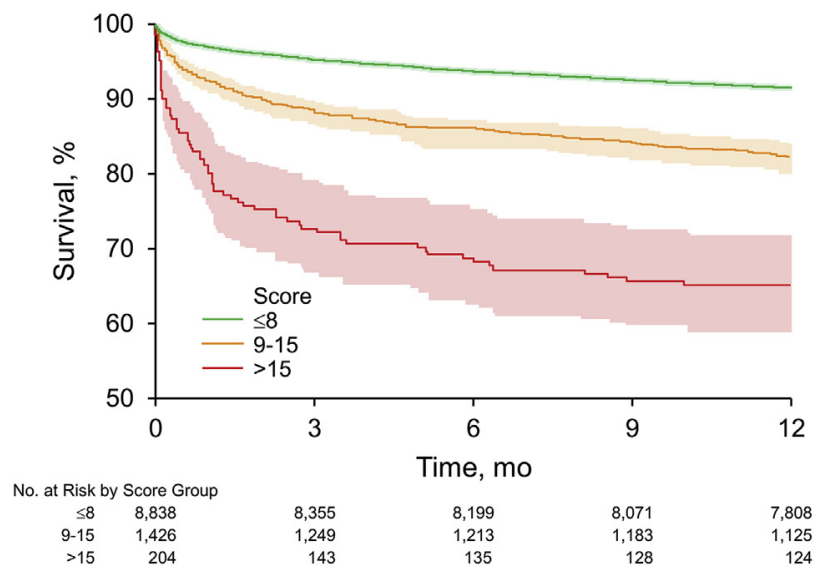

FIGURE 2. Kaplan-Meier estimates of 1-year survival after heart transplant $(P<.001)$. 


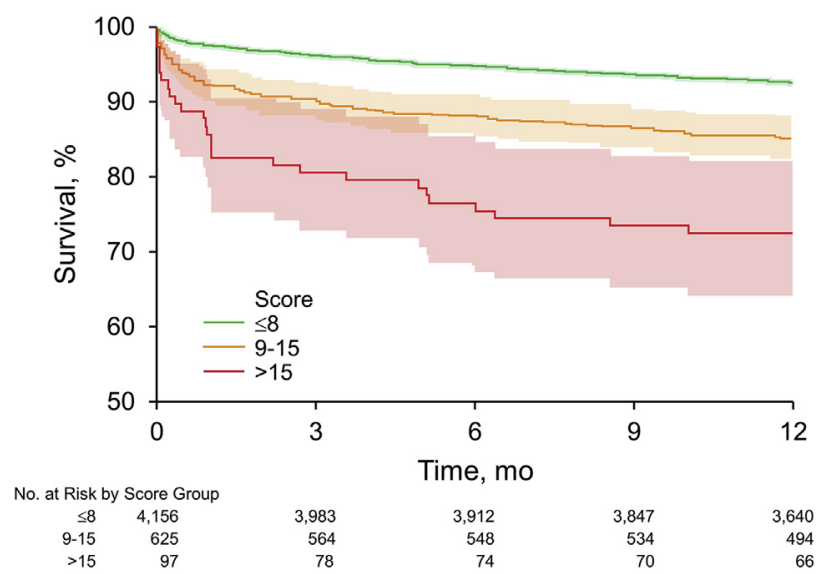

FIGURE 3. Kaplan-Meier estimates of 1-year survival of patients who underwent heart transplant after $2008(P<.001)$.

model. Furthermore, there may be additional variables that are not currently appreciated or quantifiable in the UNOS data set that could improve the $\mathrm{C}$ index in future updates to our scoring system. Lastly, the rounding process of creating the score could also cause loss in the predictive accuracy.

Another limitation from a statistical perspective derives from the fact that the proportional hazard assumption was not quite met when we used ECMO and ischemic time to predict 1-year survival. Rather than staying constant, the hazard ratios of both factors were greater in the first month of follow-up than in subsequent months. Given the challenges of incorporating these time-dependent coefficients into a scoring system, we had to use an "average effect" for these variables to predict 1-year survival. Nevertheless, it was encouraging to note that despite the rapidly changing transplant landscape, our model performed accurately in

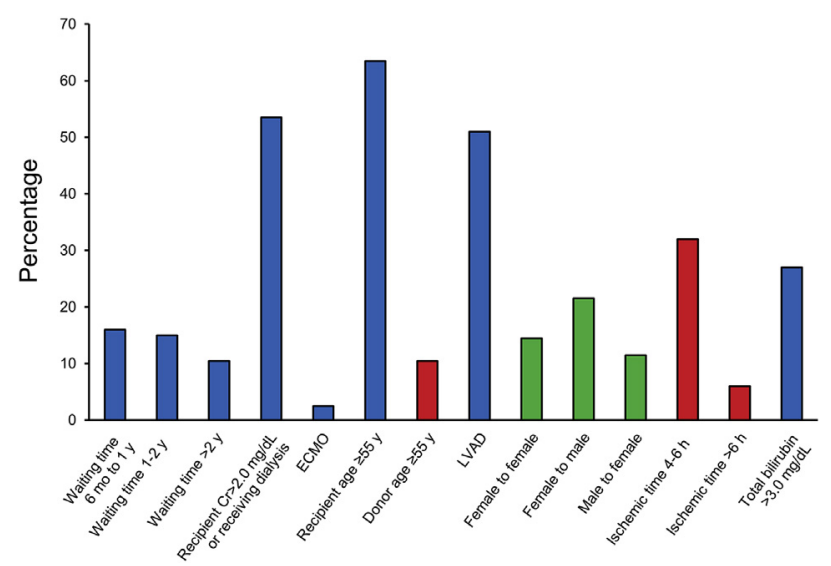

FIGURE 4. Frequency of risk factors as a percentage of the overall highrisk group. Red bars indicate donor factors; blue bars, recipient factors; and green bars, combined factors. $\mathrm{Cr}$, Creatinine; $E C M O$, extracorporeal membrane oxygenation; $L V A D$, left ventricular assist device.

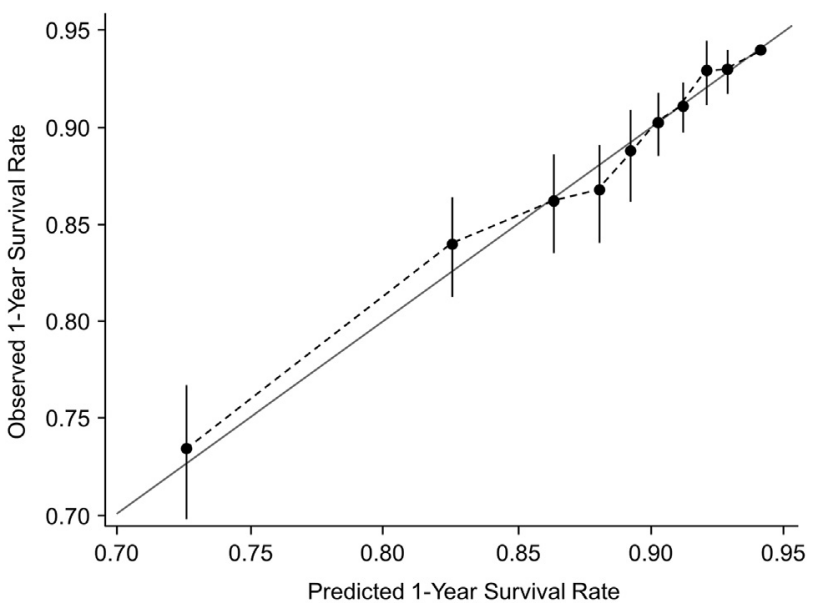

FIGURE 5. Calibration plot of observed versus predicted 1-year survival for validation population. Patients in the validation set were divided into 10 groups according to the score-predicted 1-year survival. Observed 1-year survival was plotted against the predicted survival for each group. Dots on the dashed line indicate perfect agreement between the observed and predicted survival. Solid diagonal line indicates ideal calibration plot. Whiskers indicate observed calibration plot.

stratifying risk even within the most recent subgroup analysis.

In summary, we have developed a novel approach to donor heart selection that accounts for donor, recipient, and combined donor-recipient risk factors and assesses the overall probability of a successful result (defined as survival at 1 year). We think that incorporating this type of evidence-based assessment tool will improve organ allocation efforts and refine the decisions that surround this complex process (Video 1).

\section{Conflict of Interest Statement}

Authors have nothing to disclose with regard to commercial support.

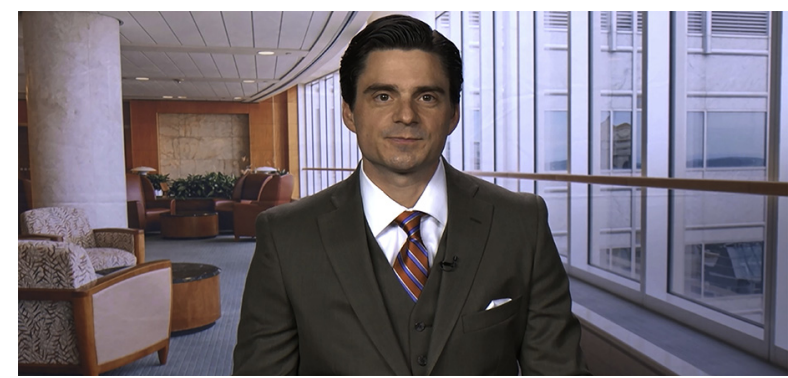

VIDEO 1. Summary of the background, methods, results, and conclusions from this analysis. (Used with permission from the Mayo Foundation for Medical Education and Research.) Video available at: http://www. jtcvsonline.org/article/S0022-5223(17)32413-3/fulltext. 


\section{References}

1. Centers for Medicare \& Medicaid Services (CMS), HHS. Medicare program hospital conditions of participation: requirements for approval and re-approval of transplant centers to perform organ transplants. Final rule. Fed Regist. 2007; 72:15197-280.

2. Kransdorf EP, Stehlik J. Donor evaluation in heart transplantation: the end of the beginning. J Heart Lung Transplant. 2014;33:1105-13.

3. Zaroff JG, Rosengard BR, Armstrong WF, Babcock WD, D'Alessandro A, Dec GW, et al. Consensus conference report: maximizing use of organs recovered from the cadaver donor: cardiac recommendations, March 28-29, 2001, Crystal City, Va. Circulation. 2002;106:836-41.

4. Stehlik J, Feldman DS, Brown RN, VanBakel AB, Russel SD, Ewald GA, et al Cardiac Transplant Research Database Group. Interactions among donor characteristics influence post-transplant survival: a multi-institutional analysis. J Heart Lung Transplant. 2010;29:291-8.

5. Weiss ES, Allen JG, Arnaoutakis GJ, George TJ, Russell SD, Shah AS, et al. Creation of a quantitative recipient risk Index for Mortality Prediction After Cardiac Transplantation (IMPACT). Ann Thorac Surg. 2011;92: 914-21.

6. Smits JM, De Pauw M, de Vries E, Rahmel A, Meiser B, Laufer G, et al. Donor scoring system for heart transplantation and the impact on patient survival. J Heart Lung Transplant. 2012;31:387-97.

7. Weiss ES, Allen JG, Kilic A, Russell SD, Baumgartner WA, Conte JV, et al Development of a quantitative donor risk index to predict short-term mortality in orthotopic heart transplantation. J Heart Lung Transplant. 2012:31:266-73.

8. Shuhaiber JH, Moore J, Dyke DB. The effect of transplant center volume on survival after heart transplantation: a multicenter study. J Thorac Cardiovasc Surg. 2010;139:1064-9.

9. Belli E, Leoni Moreno JC, Hosenpud J, Rawal B, Landolfo K. Preoperative risk factors predict survival following cardiac retransplantation: analysis of the United Network for Organ Sharing database. J Thorac Cardiovasc Surg. 2014; 147:1972-7.

10. Arnaoutakis GJ, George TJ, Allen JG, Russell SD, Shah AS, Conte JV, et al. Institutional volume and the effect of recipient risk on short-term mortality after orthotopic heart transplant. J Thorac Cardiovasc Surg. 2012;143:157-67.
11. Khush KK, Menza R, Nguyen J, Zaroff JG, Goldstein BA. Donor predictors of allograft use and recipient outcomes after heart transplantation. Circ Heart Fail. 2013;6:300-9.

12. Russo MJ, Chen JM, Sorabella RA, Martens TP, Garrido M, Davies RR, et al. The effect of ischemic time on survival after heart transplantation varies by donor age: an analysis of the United Network for Organ Sharing database. J Thorac Cardiovasc Surg. 2007;133:554-9.

13. Weber DJ, Wang IW, Gracon AS, Hellman YM, Hormuth DA, Wozniak TC, et al. Impact of donor age on survival after heart transplantation: an analysis of the United Network for Organ Sharing (UNOS) registry. J Card Surg. 2014;29: 723-8.

14. Kilic A, Allen JG, Weiss ES. Validation of the United States-derived Index for Mortality Prediction After Cardiac Transplantation (IMPACT) using international registry data. J Heart Lung Transplant. 2013;32:492-8.

15. Kirklin JK, Naftel DC, Pagani FD, Kormos RL, Stevenson LW, Blume ED, et al. Seventh INTERMACS annual report: 15,000 patients and counting. J Heart Lung Transplant. 2015;34:1495-504.

16. Al-Khaldi A, Oyer PE, Robbins RC. Outcome analysis of donor gender in heart transplantation. J Heart Lung Transplant. 2006;25:461-8.

17. Khush KK, Kubo JT, Desai M. Influence of donor and recipient sex mismatch on heart transplant outcomes: analysis of the International Society for Heart and Lung Transplantation Registry. J Heart Lung Transplant. 2012; 31:459-66.

18. Izquierdo MT, Almenar L, Martinez-Dolz L, Moro J, Aguero J, SanchezLazaro I, et al. Analysis of the impact of donor gender on early mortality. Transplant Proc. 2007;39:2375-6.

19. Michel S, Meiser B, Kaczmarek I. Impact of donor and recipient sex on outcome. Curr Opin Organ Transplant. 2011;16:543-7.

20. Rao PS, Schaubel DE, Guidinger MK, Andreoni KA, Wolfe RA, Merion RM, et al. A comprehensive risk quantification score for deceased donor kidneys: the kidney donor risk index. Transplantation. 2009;88:231-6.

Key Words: cardiac transplantation, donor selection, 1-year survival, risk-assessment tool 